\title{
An Abstract: Feel the Grip and Smell the Freedom: Assessing the Impact of Sensory Packaging on Implicit and Explicit Brand Knowledge
}

\author{
Sascha Langner, Steffen Schmidt, Gesa Lischka, Evmorfia Karampournioti, \\ and Levke Albertsen
}

\begin{abstract}
Over the last decades, there has been a significant change in packaging design from mainly informative elements toward sensorial engaging products. Consumers fundamentally experience their world through their five senses, which shape their consumption experience and affect product and brand perception and behavior. Past research has shown that packaging has an enormous influence on consumer perceptions, purchase decisions, and product acceptance. Additionally, a large amount of studies revealed valuable insights regarding how to reach consumers through the five senses. However, literature still lacks an advanced measurement approach that evaluates the impact of sensory packaging on explicit and implicit information processing in consumer's mind. The current research aims to contribute to the literature by analyzing the effect of sensory packaging on explicit and implicit product brand associations. Specifically, a PoS contact with the packaging of a premium-priced bicycle tire was simulated in a lab study to test the effectiveness of sensory packaging on consumer's brand-related association formation. Our results show that there are not only major differences between explicit and implicit sensory perception but also that it needs the right interplay of sense stimulation (here: "direct tire touch and forest scent"), in order to trigger the best brand-related association change.
\end{abstract}

\footnotetext{
S. Langner $(\bowtie) \cdot$ S. Schmidt $\cdot$ E. Karampournioti $\cdot$ L. Albertsen Leibniz University of Hannover, Hannover, Germany e-mail: langner@m2.uni-hannover.de; schmidt@m2.uni-hannover.de; karampournioti@m2.uni-hannover.de; albertsen@m2.uni-hannover.de

G. Lischka

Kochstrasse GmbH, Hannover, Germany

e-mail: lischka@kochstrasse.de
} 